\title{
Editorial:
}

\section{Frontiers in auditory bioscience and technology: a special feature on recent advances in hearing research}

\author{
Yen-Fu CHENG ${ }^{1,2,3,4,5,6}$ \\ ${ }^{I}$ Department of Otology and Laryngology, Harvard Medical School, \\ Boston, MA 02115, USA \\ ${ }^{2}$ Eaton-Peabody Laboratory, Massachusetts Eye and Ear Infirmary, \\ Boston, MA 02114, USA \\ ${ }^{3}$ Department of Medical Research, Taipei Veterans General Hospital, \\ Taipei 112, Taiwan, China \\ ${ }^{4}$ Department of Otolaryngology-Head and Neck Surgery, Taipei \\ Veterans General Hospital, Taipei 112, Taiwan, China \\ ${ }^{5}$ School of Medicine, Yang-Ming University, Taipei 112, Taiwan, \\ China \\ ${ }^{6}$ Department of Speech Language Pathology and Audiology, Taipei \\ University of Nursing and Health Science, Taipei 112, Taiwan, China \\ E-mail: yfcheng2@vghtpe.gov.tw
}

https://doi.org/10.1631/jzus.B1910001

I was greatly honored to be asked by Helen ZHANG, Former Managing Editor, to serve as a guest editor of this special feature of Journal of Zhejiang University-SCIENCE B (Biomedicine \& Biotechnology) on "Frontiers in Auditory Bioscience and Technology (FABT)".

Hearing impairment has become one of the most common sensory disabilities. The World Health Organization (WHO) estimates that 466 million people were living with disabling hearing loss in 2018, and that number could rise to 900 million by 2050 . Conductive hearing loss, which predominantly involves the sound-transmitting route of the outer and middle ear, has been well handled by antibiotics and surgery. However, sensorineural hearing loss, which involves the inner ear and structures further within the auditory pathway, has very limited biological treatment options (current treatment options include only hearing

(1D ORCID: Yen-Fu CHENG, https://orcid.org/0000-0003-1995-5854 (C) Zhejiang University and Springer-Verlag GmbH Germany, part of Springer Nature 2019 amplification and cochlear implants). Part of the reason for the paucity of therapeutics is due to the complexity of the auditory system and the limited regenerative ability of the hearing sensory cells, hair cells, and connected nerve.

With recent progress in molecular biology and genetic medicine, researchers have started to dig deeper into the mechanisms underlying hearing loss. Based on these studies, more potential treatment options are under investigation.

In this special feature, we were lucky to recruit scientists who are active in the hearing field to share their perspectives and research. The first article by Mizutari (2019) reviews recent studies of blastinduced hearing dysfunction. He outlines the current understanding of conductive and sensorineural pathologies underlying blast-induced hearing loss, as well as some recent progress in the research. He also stresses the further need for studies of the detailed mechanisms and new treatment options.

In the next article, Peter and Kleinjung (2019) review the current neuromodulation techniques for the treatment of tinnitus. The authors provide extended perspective and discussion on the use and limitations of current non-invasive and invasive neuromodulation methods for the emerging field of hearing medicine.

The next two articles examine the molecular signals that regulate inner ear development and regeneration. Ma et al. (2019) review the role of bone morphogenetic proteins (BMPs) and the crosstalk between BMP signaling and other signaling pathways in the development of the inner ear. Next, Cheng (2019) discusses the role of the basic helix-loop-helix (bHLH) transcription factor Atoh1 and its transcriptional and posttranslational regulation during inner ear development. Both articles hope to provide a better understanding of cochlear development and, 
thus, offer more insight into therapeutic strategies for hair cell regeneration in the future.

Finally, Wang et al. (2019) present a clinical study of otogenic cerebrospinal fluid leakage secondary to congenital inner ear dysplasia. They also discuss the diagnosis and treatment of this rare and challenging issue. Zheng et al. (2019) then report new single nucleotide polymorphism (SNP) variants of a gene associated with nonsyndromic hearing loss (DFNB49) in the Chinese population.

These inspiring examples are a sampling of the recent work addressing significant progress in both clinical and basic hearing research. We are excited that the understanding and treatment of hearing disorders is heading into a new era. We are delighted to present this special feature of recent progress toward these goals.

\section{Compliance with ethics guidelines}

Yen-Fu CHENG declares that he has no conflict of interest.

This article does not contain any studies with human or animal subjects performed by the author.

\section{References}

Cheng YF, 2019. Atoh1 regulation in the cochlea: more than just transcription. J Zhejiang Univ-Sci B (Biomed \& Biotechnol), 20(2):146-155.

https://doi.org/10.1631/jzus.B1600438

Ma JY, You D, Li WY, et al., 2019. Bone morphogenetic proteins and inner ear development. $J$ Zhejiang Univ-Sci B (Biomed \& Biotechnol), 20(2):131-145. https://doi.org/10.1631/jzus.B1800084

Mizutari KJ, 2019. Blast-induced hearing loss. J Zhejiang Univ-Sci B (Biomed \& Biotechnol), 20(2):111-115. https://doi.org/10.1631/jzus.B1700051

Peter N, Kleinjung TJ, 2019. Neuromodulation for tinnitus treatment: an overview of invasive and non-invasive techniques. J Zhejiang Univ-Sci B (Biomed \& Biotechnol), 20(2):116-130. https://doi.org/10.1631/jzus.B1700117

Wang B, Dai WJ, Cheng XT, et al., 2019. Cerebrospinal fluid otorrhea secondary to congenital inner ear dysplasia: diagnosis and management of 18 cases. J Zhejiang Univ-Sci B (Biomed \& Biotechnol), 20(2):156-163. https://doi.org/10.1631/jzus.B1800224

Zheng J, Meng W, Zhang C, et al., 2019. New SNP variants of MARVELD2 (DFNB49) associated with non-syndromic hearing loss in Chinese population. J Zhejiang Univ-Sci B (Biomed \& Biotechnol), 20(2):164-169. https://doi.org/10.1631/jzus.B1700185 